\title{
Towards a classification of maximal peak-pit Condorcet domains
}

by Guanhao Li, Clemens Puppe and Arkadii Slinko

No. 144 | SEPTEMBER 2020

\section{WORKING PAPER SERIES IN ECONOMICS}

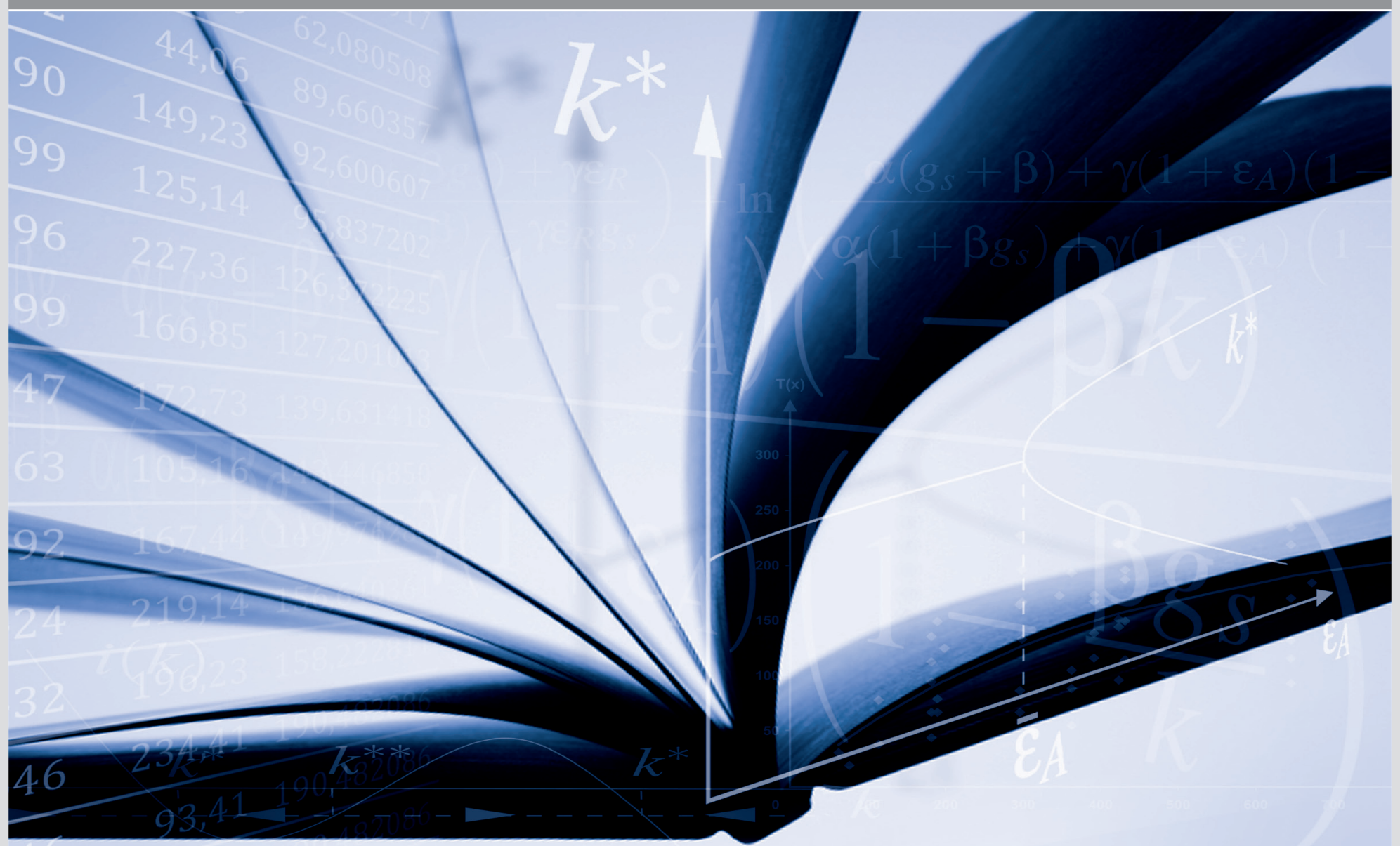




\section{Impressum}

Karlsruher Institut für Technologie (KIT)

Fakultät für Wirtschaftswissenschaften

Institut für Volkswirtschaftslehre (ECON)

Kaiserstraße 12

76131 Karlsruhe

KIT - Die Forschungsuniversität in der Helmholtz-Gemeinschaft

Working Paper Series in Economics

No. 144, September 2020

ISSN 2190-9806

econpapers.wiwi.kit.edu 


\title{
Towards a classification of maximal peak-pit Condorcet domains
}

\author{
Guanhao $\mathrm{Li}^{1}$, Clemens Puppe ${ }^{2}$, and Arkadii Slinko ${ }^{3}$ \\ ${ }^{1,3}$ Department of Mathematics, University of Auckland (New Zealand) \\ ${ }^{2}$ Department of Economics and Management, Karlsruhe Institute of Technology (Germany) \\ and Higher School of Economics (Russian Federation)
}

September 2020

\begin{abstract}
In this paper, we classify all maximal peak-pit Condorcet domains of maximal width for $n \leq 5$ alternatives. To achieve this, we bring together ideas from several branches of combinatorics. The main tool used in the classification is the ideal of a domain. In contrast to the size of maximal peak-pit Condorcet domains of maximal width themselves, the size of their associated ideal is constant.
\end{abstract}

\section{Introduction}

Condorcet domains are sets of linear orders such that the pairwise majority relation is acyclic whenever all individuals have preferences taken from the given set; they are therefore sometimes also referred to as acyclic sets of linear orders [Fishburn, 1996] Condorcet domains play an important role in applications because they allow both for Arrovian aggregation [Aleskerov, 1999] and for non-manipulable social choice [Puppe and Slinko, 2019]; well-known examples are the single-peaked and the single-crossing domains.

A major research question in the literature has been the problem of describing 'large' Condorcet domains. It is well-known that on a set of $n$ alternatives there always exist Condorcet domains of cardinality $2^{n-1}$, indeed this cardinality is achieved by the domain of all preferences that are single-peaked with respect to a given linear ordering of the underlying set of alternatives; however, it is also known that, except for the case $n \leq 3$, this is not the largest cardinality of a Condorcet domain, see Monjardet [2009]. In the search for large Condorcet domains, the class of so-called 'peak-pit' Condorcet domains of maximal width have been a main object of investigation, and in fact members of this class have been shown to constitute the Condorcet domains of maximal cardinality for 
$n \leq 6 .{ }^{1}$. Peak-pit domains are also distinguished by a number of deep connections to other combinatorial concepts such as reduced decompositions of permutations, rhombus tilings and arrangements of pseudolines [Galambos and Reiner, 2008, Danilov et al., 2012].

The concept of the ideal of a domain of linear orders was introduced in Danilov et al. [2012] as a generalization of the spectrum of a rhombus tiling and the family of chamber sets of an arrangement of pseudolines. It was used as a key technical tool in proving that the class of maximal peak-pit domains of maximal width coincides with the class of rhombus tiling domains. Specifically, Danilov et al. [2012] established that the ideal of a peak-pit domain is a separated system of subsets; ${ }^{2}$ this allowed them to use (i) the result of Leclerc and Zelevinsky [1998] who proved that maximal separated systems of sets are exactly the set of labels of chambers of arrangements of pseudolines, and (ii) the correspondence between rhombus tilings and arrangements of pseudolines established in Elnitsky [1997].

In this paper, we show how the method of ideals can be principally applied to the classification of all maximal peak-pit domains of maximal width, and we carry the steps out for alternative sets with $n \leq 5$ alternatives. Up to isomorphism and flip-isomorphism, there are 18 maximal Condorcet domains on a set of four alternatives, and 688 maximal Condorcet domains on a set of five alternatives [Dittrich, 2018]. For $n=4$, only three of the 18 maximal Condorcet domains are peak-pit domains of maximal width: a single-crossing domain, the single-peaked domain, and the domain obtained by Fishburn's alternating scheme; these domains have cardinalities 7, 8 and 9 , respectively. For $n=5$, we show that among the 688 maximal Condorcet domains there are exactly 18 peak-pit domain of maximal width and we list them explicitly; the possible cardinalities of these domains are 11, 12, 14, 15, 16, 17, 19 and 20.

A number of important properties of peak-pit domains of maximal width follow directly or indirectly from their connections to the aforementioned combinatorial concepts. One of the most important is that every maximal peak-pit domain of maximal width is semiconnected, that is, the two completely reversed orders can be connected by a shortest path in the permutahedron, see Danilov et al. [2012], Puppe and Slinko [2019]. We strengthen this result by showing that any two linear orders of a maximal peak-pit Condorcet domain of maximal width are in fact connected by a geodesic path in the permutahedron. We also show that the cardinality of the ideal of every maximal peak-pit domain of maximal width on $n$ alternatives is exactly $\left(\begin{array}{c}n+1 \\ 2\end{array}\right)+1$. This is all the more remarkable since the cardinality of maximal peak-pit domains itself can vary significantly.

\footnotetext{
${ }^{1}$ Not much is known about the structure of Condorcet domains of maximal cardinality for $n>6$; however, the peak-pit Condorcet domains of maximal width do not always achieve the maximal cardinality [Fishburn, 1996]

${ }^{2}$ In Leclerc and Zelevinsky [1998] such systems are called 'strongly' separated.
} 


\section{Condorcet domains and their combinatorial repre- sentations}

\subsection{Basics of Condorcet domains}

Let $A$ be a finite set and $\mathcal{L}(A)$ be the set of all (strict) linear orders on $A$. Any subset $\mathcal{D} \subseteq \mathcal{L}(A)$ will be called a domain. Any sequence $P=\left(v_{1}, \ldots, v_{n}\right)$ of linear orders from $\mathcal{D}$ will be called a profile over $\mathcal{D}$. If $a_{1}>a_{2}>\cdots>a_{n}$ is a linear order on $A$, it will be denoted by a string $a_{1} a_{2} \ldots a_{n}$. Let us also introduce notation for reversing orders, i.e., if $v=a_{1} a_{2} \ldots a_{n}$, then $\bar{v}=a_{n} a_{n-1} \ldots a_{1}$. We will also write $a>_{v} b$ if $a$ is rated higher than $b$ in the linear order $v$.

Definition 1. The majority relation $\succeq_{P}$ of any profile $P=\left(\succ_{1}, \ldots, \succ_{n}\right)$ is defined as

$$
a \succeq_{P} b \Longleftrightarrow\left|\left\{i \mid a \succ_{i} b\right\}\right| \geq\left|\left\{i \mid b \succ_{i} a\right\}\right| \text {. }
$$

For an odd number of linear orders in the profile $P$ this relation is a tournament, i.e., complete and asymmetric binary relation. In this case we denote it $\succ_{P}$.

Now we can define the main object of this investigation.

Definition 2. A domain $\mathcal{D} \subseteq \mathcal{L}(A)$ over a set of alternatives $A$ is a Condorcet domain if the majority relation of any profile $P$ over $\mathcal{D}$ is transitive. A Condorcet domain $\mathcal{D}$ is maximal if for any Condorcet domain $\mathcal{D}^{\prime} \subseteq \mathcal{L}(A)$ the inclusion $\mathcal{D} \subseteq \mathcal{D}^{\prime}$ implies $\mathcal{D}=\mathcal{D}^{\prime}$.

Let $\psi: A \rightarrow A^{\prime}$ be a bijection between two sets of alternatives. It can be extended to a mapping $\psi: \mathcal{L}(A) \rightarrow \mathcal{L}\left(A^{\prime}\right)$ in two ways: by mapping linear order $u=a_{1} a_{2} \ldots a_{m}$ onto $\psi(u)=\psi\left(a_{1}\right) \psi\left(a_{2}\right) \ldots \psi\left(a_{m}\right)^{3}$ or to $\overline{\psi(u)}=\psi\left(a_{m}\right) \psi\left(a_{m-1}\right) \ldots \psi\left(a_{1}\right)$.

Definition 3. Let $A$ and $A^{\prime}$ be two sets of alternatives (not necessarily distinct) of equal cardinality $m$. We say that two domains, $\mathcal{D} \subseteq \mathcal{L}(A)$ and $\mathcal{D}^{\prime} \subseteq \mathcal{L}\left(A^{\prime}\right)$ are isomorphic if there is a bijection $\psi: A \rightarrow A^{\prime}$ such that $\mathcal{D}^{\prime}=\{\psi(d) \mid d \in \overline{\mathcal{D}}\}$ and flip-isomorphic if $\mathcal{D}^{\prime}=\{\overline{\psi(d)} \mid d \in \mathcal{D}\}$.

In the particular case, when $\overline{\mathcal{D}}=\{\bar{u} \mid u \in \mathcal{D}\}$, we call the domain $\overline{\mathcal{D}}$ as fipped $\mathcal{D}$.

Up to an isomorphism, there is only one maximal Condorcet domain on the set $\{a, b\}$, namely $C D_{2}=\{a b, b a\}$ and there are are only three maximal Condorcet domains on the set of alternatives $\{a, b, c\}$, namely,

$$
C D_{3, t}=\{a b c, a c b, c a b, c b a\}, C D_{3, m}=\{a b c, b c a, a c b, c b a\}, C D_{3, b}=\{a b c, b a c, b c a, c b a\} .
$$

No two of them are isomorphic but $C D_{3, t}$ and $C D_{3, b}$ are flip-isomorphic under the identity mapping of $\{a, b, c\}$ onto itself. The first domain contains all the linear orders on $\{a, b, c\}$ where $b$ is never ranked first, second contains all the linear orders on $\{a, b, c\}$ where $a$ is never ranked second and the third contains all the linear orders on $\{a, b, c\}$ where $b$ is

\footnotetext{
${ }^{3}$ We use the same notation for both mappings since there can be no confusion.
} 
never ranked last. Following Monjardet [2009], we denote these conditions as $b N_{\{a, b, c\}} 1$, $a N_{\{a, b, c\}} 2$ and $b N_{\{a, b, c\}} 3$, respectively. We note that these are the only conditions of type $x N_{\{a, b, c\}} i$ with $x \in\{a, b, c\}$ and $i \in\{1,2,3\}$ that these domains satisfy.

In general, given any set of alternatives $A$, we say that

$$
\mathcal{N}=\left\{x N_{\{a, b, c\}} i \mid\{a, b, c\} \subseteq A, x \in\{a, b, c\} \text { and } i \in\{1,2,3\}\right\}
$$

is a complete set of never conditions if it contains at least one never condition for every triple $\{a, b, c\}$ of distinct elements of $A$. If the set of linear orders that satisfy $\mathcal{N}$ is non-empty, we say that $\mathcal{N}$ is consistent. ${ }^{4}$ There are a number of alternative characterizations of Condorcet domains [Monjardet, 2009, Puppe and Slinko, 2019]; the following is particularly useful for our purposes.

Proposition 1. A domain of linear orders $\mathcal{D} \subseteq \mathcal{L}(A)$ is a Condorcet domain if and only if it is non-empty and satisfies a complete set of never conditions.

Proof. This is a well-known characterization which goes back to Sen [1966]. See also Theorem 1(d) in Puppe and Slinko [2019] and references therein.

This proposition in particular means that the collection $\mathcal{D}(\mathcal{N})$ of all linear orders that satisfy a certain complete set of never conditions $\mathcal{N}$, if non-empty, is a Condorcet domain. Let us also denote by $\mathcal{N}(\mathcal{D})$ the set of all never conditions that are satisfied by all linear orders from $\mathcal{D}$.

Definition 4. A Condorcet domain $\mathcal{D}$ is said to have maximal width if together with some linear order $u$ it also contains $\bar{u}$.

Up to an isomorphism, for any Condorcet domain of maximal width we may assume that it contains orders $e=12 \ldots n$ and $\bar{e}=n \ldots 21 .^{5}$ The property of maximal width plays an important and role in the analysis of Condorcet domains and simplifies matters sometimes considerably. This is also reflected by the fact that a maximal Condorcet domain can be naturally endowed with the structure of a distributive lattice if and only if it has maximal width, see Corollary 3.2 in Puppe and Slinko [2019] and the references there.

A domain that, for any triple $\{a, b, c\} \subseteq A$, satisfies a condition $x N_{\{a, b, c\}} 1$ with $x \in$ $\{a, b, c\}$ is called never-top domain, a domain that for any triple $\{a, b, c\} \subseteq A$ satisfies a condition $x N_{\{a, b, c\}} 2$ with $x \in\{a, b, c\}$ is called never-middle domain, and a domain that for any triple $\{a, b, c\} \subseteq A$ satisfies a condition $x N_{\{a, b, c\}} 3$ with $x \in\{a, b, c\}$ is called neverbottom domain. A domain that for any triple satisfies either never-top or never-bottom condition is called peak-pit domain [Danilov et al., 2012]. Both never-top and neverbottom conditions will be called peak-pit conditions. All peak-pit domains with maximal width can be embedded into the so-called 'tiling' domains, see Theorem 2 of Danilov et al. [2012]. The never-bottom domains are also known as Arrow's single-peaked domains;

\footnotetext{
${ }^{4}$ It is easy to construct inconsistent sets of never conditions; for example, requiring that no alternative is ever listed last in a given triple is evidently inconsistent.

${ }^{5}$ Danilov and Koshevoy [2013] call such domains 'normal.'
} 
they have been characterized by Slinko [2019]. The Arrow single-peaked domains with maximal width correspond to the standard ('Black's') single-peaked domains and have been characterized by Puppe [2018].

The universal domain $\mathcal{L}(A)$ is naturally endowed with the following betweenness structure [Kemeny, 1959]. An order $v$ is between orders $u$ and $w$ if $v \supseteq u \cap w$, i.e. if $v$ agrees with all binary comparisons in which $u$ and $w$ agree (see also Kemeny and Snell [1960]). The set of all orders that are between $u$ and $w$ is called the interval spanned by $u$ and $w$ and is denoted by $[u, w]$. The domain $\mathcal{L}(A)$ endowed with this betweenness relation is referred to as the permutahedron [Monjardet, 2009].

Given a domain of preferences $\mathcal{D}$, for any $u, w \in \mathcal{D}$ we define the induced interval as $[u, w]_{\mathcal{D}}=[u, w] \cap \mathcal{D}$. Puppe and Slinko [2019] defined a graph $G_{\mathcal{D}}$ associated with $\mathcal{D}$ as follows. The set of linear orders from $\mathcal{D}$ is the set of vertices $V_{\mathcal{D}}$ of $G_{\mathcal{D}}$, and for two orders $u, w \in \mathcal{D}$ we draw an edge between them if there is no other vertex between them, i.e., if $[u, w]_{\mathcal{D}}=\{u, w\}$ (i.e., if $u$ and $w$ are 'neighbors' in $\mathcal{D}$ ). The set of edges is denoted $E_{\mathcal{D}}$ so the graph is $G_{\mathcal{D}}=\left(V_{\mathcal{D}}, E_{\mathcal{D}}\right)$. As established in Puppe and Slinko [2019], for every Condorcet domain $\mathcal{D}$ the graph $G_{\mathcal{D}}$ is a median graph [Mulder, 1978] and any median graph can be obtained in this way.

Besides the single-crossing and the single-peaked domains, the best-known peak-pit domains are Fishburn's domains obtained by the so-called alternating scheme.

Definition 5. A complete set of never-conditions (1) is said to satisfy the alternating scheme, if for all $1 \leq i<j<k \leq n$ either

(i) $j N_{\{i, j, k\}} 1$, if $j$ is even, and $j N_{\{i, j, k\}} 3$, if $j$ is odd, or

(ii) $j N_{\{i, j, k\}} 3$, if $j$ is even, and $j N_{\{i, j, k\}} 1$, if $j$ is odd.

Following Galambos and Reiner [2008] we denote these domains as $F_{n}$ and $\overline{F_{n}}$. The second domain is flip-isomorphic to the first. In particular, $F_{2}=\{12,21\}, F_{3}=\{123,132,312,321\}$ and

$$
F_{4}=\{1234,1324,3124,1342,3142,3412,4312,3421,4321\} .
$$

The latter has the following median graph associated with it:

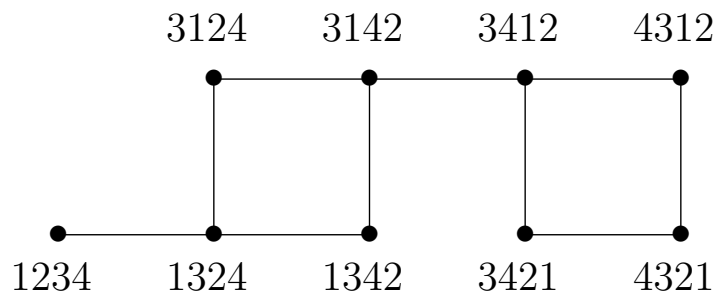

Figure 1: Graph of Fishburn's domain $F_{4}$ on four alternatives

Note that $F_{4}$ has cardinality 9 and is in fact the (uniquely) largest Condorcet domain on a set of four alternatives. 
Definition 6 (Danilov et al. [2012]). A domain $\mathcal{D}$ of maximal width is called semiconnected if the two completely reversed orders e and $\bar{e}$ can be connected by a shortest path (geodesic path) in the permutahedron whose all vertices belong to $\mathcal{D}$.

Danilov et al. [2012] proved that a maximal Condorcet domain is semi-connected if and only if it is a peak-pit domain with maximal width. To achieve this they established a one-to-one correspondence between maximal peak-pit domains of maximal width and rhombus tilings. Danilov et al. [2012] also showed that peak-pit domains with maximal width are in fact connected in the following stronger sense. (There are different notions of connectedness in the literature.)

Definition 7. We call a domain $\mathcal{D}$ of linear orders connected if it is a connected subgraph of the permutahedron.

Below, we will prove that peak-pit domains with maximal width indeed satisfy the following even stronger notion of connectedness, see Puppe [2016].

Definition 8. We call a domain $\mathcal{D}$ of linear orders directly connected if any two orders of this domain can be connected by a shortest path in the permutahedron whose all vertices are in $\mathcal{D}$.

Clearly, any directly connected Condorcet domain is connected and semi-connected (if of maximal width). For example, the domains $C D_{3, t}$ and $C D_{3, b}$ above are directly connected, but $C D_{3, m}$ is not even connected or semi-connected.

Our final definition concerns the number different restrictions that a Condorcet domain induces on triples, see Slinko [2019].

Definition 9. A Condorcet domain $\mathcal{D}$ is called copious if for any triple of alternatives $\{a, b, c\} \subseteq A$ the restriction $\mathcal{D}_{\{a, b, c\}}$ of this domain to the triple has four distinct orders, that is, $\left|\mathcal{D}_{\{a, b, c\}}\right|=4$.

We note that, if a Condorcet domain is copious, then it satisfies a unique complete set of never conditions of the form (1).

In a semi-connected domain the order $12 \ldots n$ can be transformed into $n \ldots 21$ by a sequence of swaps of neighboring alternatives. If $i<j<k$, then there are two ways of converting $i j k$ into $k j i$, namely,

$$
i j k \rightarrow j i k \rightarrow j k i \rightarrow k j i \quad \text { and } \quad i j k \rightarrow i k j \rightarrow k i j \rightarrow k j i .
$$

In the second case the triple $[i, j, k]$ is called an inversion. The inversion triples characterize maximal semi-connected Condorcet domains uniquely [Galambos and Reiner, 2008] and provide a convenient description of these domains.

\subsection{Arrangements of pseudolines and separated collections of subsets}

In this section we will describe a geometric representation of reduced decompositions by pseudoline arrangements on the plane. This representation has by now become folklore in low dimensional topology, the study of the Yang-Baxter equation, and geometric 
combinatorics [Humphreys, 1994]. Galambos and Reiner [2008] related these concepts to Condorcet domains.

The most intuitive way to think about an arrangements of pseudolines is geometrically. On two vertical parallel lines of the same length in $\mathbb{R}^{2}$, we mark a set of $n$ equidistant points. The points on the left line are labeled $1, \ldots, n$ in downward order and on the right line the points are marked also $1 \ldots, n$ but in upward order. The two points with the same label $i$ - one on the left and one on the right — are joined by a continuous curve which is called pseudoline $i$ so that any two pseudolines intersect at most at one point, called a vertex. The arrangement is simple if there is no vertex where three or more pseudolines meet.

An arrangement of pseudolines consisting of piecewise linear 'wires' is called a wiring diagram. The wires (pseudolines) are horizontal except for small neighborhoods of their crossings with other wires; see Figure 2 for an example. There is no loss of generality in assuming that our pseudolines are wires. The arrangements we consider are all simple, and often called simple numbered arrangements of pseudolines, see [Björner et al., 1999, Sect. 6.4].

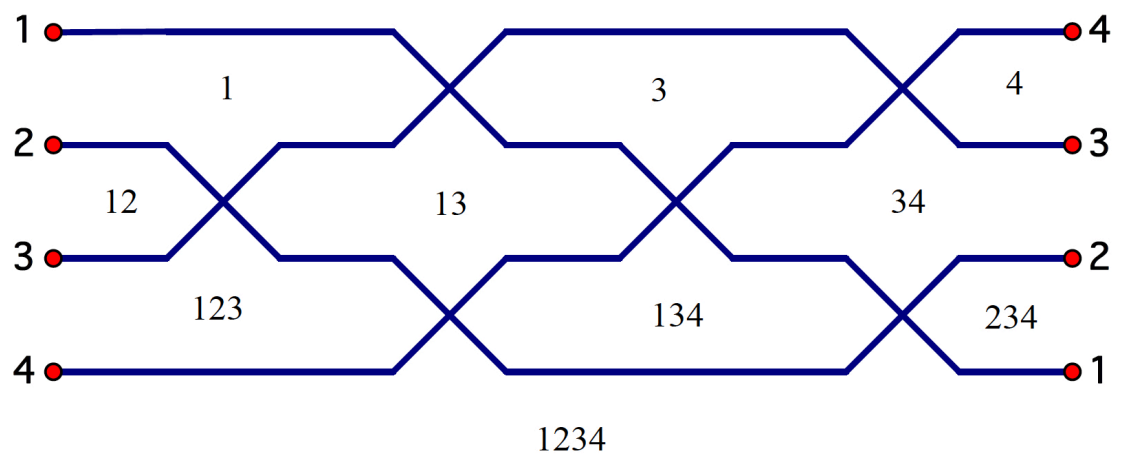

Figure 2: Example of a wiring diagram. As we will see later it is intimately related to Fishburn's domain $F_{4}$.

The vertices in a wiring diagram are situated on $n-1$ levels, we count these levels in downward order. We remind the reader that an adjacent transposition of $S_{n}$ is $s_{i}=$ $(i, i+1)$, where $1 \leq i \leq n-1$, and that all $n-1$ adjacent transpositions generate the whole permutation group $S_{n}$. The adjacent transpositions satisfy the so-called short braid relation:

$$
s_{i} s_{j}=s_{j} s_{i} \text { for all }|i-j|>1 \text {; }
$$

Permutations from $S_{n}$ act on linear orders from $\mathcal{L}([n])$, where we denote $[n]:=$ $\{1, \ldots, n\}$. If $v$ is a linear order then $v s_{i}$ interchanges the alternatives in positions $i$ and $i+1$ in $v$. That is, if $v=v_{1} \ldots v_{n}$, then $v s_{i}=v_{1} \ldots v_{i+1} v_{i} \ldots v_{n}$.

We say that $\left(i_{1}, \ldots, i_{p}\right)$ is a reduced decomposition of $w \in S_{n}$, if

$$
w=s_{i_{1}} \ldots s_{i_{p}},
$$


where $p$ is minimal, in which case

$$
p=\ell(w)=\#\left\{(i, j) \mid i<j, \text { and } w_{i}>w_{j}\right\}
$$

i.e., $p$ is the number of inversions $\operatorname{inv}(w)$ or length $\ell(w)$ of $w$. In this case $w$ can be obtained from $e=12 \ldots n$ by making swaps of adjacent alternatives $s_{i_{1}} \ldots s_{i_{p}}$. We will be especially interested in the reduced decompositions of $\bar{e}=n \ldots 21$.

Definition 10 (Galambos and Reiner [2008]). Two reduced decompositions of $\bar{e} \in S_{n}$ are equivalent if they can be transformed one into the other using only the short braid relation.

Transpositions of alternatives in positions $i$ and $i+1$ correspond to a vertex at level $n-i$ in the arrangement of pseudolines. For example, in the arrangement on Figure 2 we have $\bar{e}=(1,4)(2,3)$ which has four equivalent reduced decompositions:

$$
\bar{e}=s_{2} s_{1} s_{3} s_{2} s_{1} s_{3}=s_{2} s_{1} s_{3} s_{2} s_{3} s_{1}=s_{2} s_{3} s_{1} s_{2} s_{1} s_{3}=s_{2} s_{3} s_{1} s_{2} s_{3} s_{1} .
$$

Each action of a transposition corresponds to a particular vertex in Figure 2; for instance, the last occurrence of transposition $s_{1}$ corresponds to the right-most vertex on the top; similarly, the last occurrence of transposition $s_{3}$ corresponds to the right-most vertex at the bottom, and so on. In this manner, arrangements of pseudolines turn out to be a convenient and simple representation of equivalence classes of reduced decompositions [Grunbaum, 1972]. For a given $n$, we denote the set of all possible arrangements of pseudolines by $\mathcal{A}_{n}$.

The complement of the pseudolines in an arrangement is split into chambers which are the connected parts of this complement. They are labeled as follows. For any chamber $C$ and any pseudoline $k$ we can say if this chamber is above or below the line $k$. The label of the chamber is the set of numbers of the pseudolines that go above this chamber (see Figure 2 for an illustration). Given an arrangement of pseudolines $\mathbf{a} \in \mathcal{A}_{n}$ the set of the corresponding labels of chambers - abbreviated further as chamber set - will be denoted as $C(\mathbf{a})$. This set is naturally 'graded' in the sense that

$$
C(\mathbf{a})=\bigcup_{i=0}^{n} C_{i}(\mathbf{a}),
$$

where $C_{i}(\mathbf{a})$ is the family of chamber sets of cardinality $i$.

For example, for the arrangement of pseudolines on Figure 2 the set of chamber sets consists of

$$
\emptyset,\{1\},\{3\},\{4\},\{1,2\},\{1,3\},\{3,4\},\{1,2,3\},\{1,3,4\},\{2,3,4\},\{1,2,3,4\} .
$$

Definition 11. Two sets $X, Y \subseteq[n]$ are said to be separated if there does not exist a triple $\{a, b, c\} \subseteq[n]$ such that $a<b<c$ and

(i) $X \cap\{a, b, c\}=\{b\}$; 
(ii) $Y \cap\{a, b, c\}=\{a, c\}$.

A system of subsets $S \subseteq 2^{[n]}$ is called separated if any two sets in $S$ are separated.

For example, the sets $\{1,3,4\}$ and $\{1,4,5\}$ are separated but $\{1,3,4\}$ and $\{2,4,5\}$ are not. This definition (more convenient to us) is equivalent to the more standard definition of separability introduced in Leclerc and Zelevinsky [1998] (see their Lemma 3.7 for the equivalence).

It is easy to see that for any arrangement $\mathbf{a}$ of pseudolines the chamber $\operatorname{set} C(\mathbf{a})$ is separated. Moreover, the following theorem holds.

Theorem 1 (Leclerc and Zelevinsky [1998]). The mapping $\mathbf{a} \mapsto C(\mathbf{a})$ is a bijection between the set of arrangements of pseudolines from $\mathcal{A}_{n}$ and the sets of all maximal (by inclusion) separated collections of subsets of $[n]$.

Maximal separated collections of subsets of $[n]$ always contain some particular subsets as observed by the following result.

Proposition 2. Any maximal collection of separated subsets $S \subseteq 2^{[n]}$ contains all of the following subsets

$$
\emptyset,\{1\},\{1,2\}, \ldots,\{1,2, \ldots, n\},\{2, \ldots, n\}, \ldots,\{n-1, n\},\{n\} .
$$

Proof. Let $X=\{1,2, \ldots, k\}, Y$ be any subset of $[n]$, and $\{a, b, c\} \subseteq[n]$ such that $a<$ $b<c$. Then $X \cap\{a, b, c\}$ cannot be $\{b\}$ or $\{a, c\}$, hence $X$ and $Y$ are separated no matter what $Y$ is. Since $S$ was assumed to be maximal, $X$ is already in $S$. The argument for sets of the form $X=\{k, k+1, \ldots, n\}$ is similar.

Galambos and Reiner [2008] showed that maximal semi-connected Condorcet domains of maximal width can be obtained by collecting all permutations visited by an equivalence class of maximal reduced decompositions of $\bar{e}$. They also showed how the Condorcet domain can be reconstructed from the arrangement of pseudolines corresponding to the equivalence class of reduced decompositions. The key to this is the notion of an ideal to which we turn now.

\subsection{Separated ideals and peak-pit domains}

Let $A=\left\{a_{1}, \ldots, a_{n}\right\}$. be a finite set with $|A|=n$. Every subset $I \subseteq 2^{A}$ is graded in the sense that $I=\bigcup_{k=0}^{n} I_{k}$, where $I_{k}=\{J \in I|| J \mid=k\}$. A graded set $I \subseteq 2^{A}$ is called an ideal in $A$ if:

(I0) $I_{0}=\emptyset$;

(I1) For all $k=0,1, \ldots, n-1$, if $X \in I_{k}$, then there exist $a \in A$ such that $X \cup\{a\} \in I_{k+1}$;

(I2) For all $k=1,2, \ldots, n$, if $X \in I_{k}$, then there exist $a \in X$ such that $X \backslash\{a\} \in I_{k-1}$.

The role of ideals is revealed in the following proposition. 
Proposition 3. For any arrangement of pseudolines a the collection of chamber sets $C(\mathbf{a})$ is an ideal.

Proof. For any chamber $C$ which is not $\emptyset$ or $[n]$ there are two pseudolines, one below and one above, say $i$ and $j$, respectively, that form parts of the border of $C$. Hence both $C \backslash\{i\}$ and $C \cup\{j\}$ belong to $C(\mathbf{a})$.

From Theorem 1 and Proposition 3 we also have the following important corollary.

Proposition 4. Any maximal separated collection of subsets in $[n]$ is an ideal.

Let us now explain how domain and ideals are related.

Definition 12. We call a sequence $F=\left(F_{0}, F_{1}, \ldots, F_{n}\right)$ of subsets of $A$ a flag if for some permutation $\sigma \in S_{n}$ we have $F_{k}=\left\{a_{\sigma(1)}, \ldots, a_{\sigma(k)}\right\}$. The linear order $a_{\sigma(1)} a_{\sigma(2)} \ldots a_{\sigma(n)}$ is said to correspond to the flag $F$.

We say that a flag $F=\left(F_{0}, F_{1}, \ldots, F_{n}\right)$ belongs to an ideal $I=\bigcup_{i=0}^{n} I_{i}$ iff $F_{k} \in I_{k}$ for all $k=0,1, \ldots, n$. We write $F \in I$ to denote this.

Proposition 5. Any ideal $I$ is the union of its flags, i.e., $I_{k}=\left\{F_{k} \mid F \in I\right\}$.

Proof. It is sufficient to show that any $X \in I_{k}$ is the $k$-th component of a flag belonging to $I$. This follows from conditions (I1) and (I2).

Let us show that every domain of linear orders determines an ideal and vice versa. Let $u=x_{1} x_{2} \ldots x_{n} \in \mathcal{L}(A)$ be a linear order on $n=|A|$ alternatives. By $u_{k}=x_{1} \ldots x_{k}$ we will denote the initial segment of $u$ of length $k$. We set $\operatorname{Id}_{k}(u)=\left\{x_{1}, \ldots, x_{k}\right\}$.

Definition 13. Let $\mathcal{D}$ be any domain. By the ideal of this domain we mean the set of subsets of $A$

$$
I d(\mathcal{D}):=\bigcup_{k=0}^{n} I d_{k}(\mathcal{D}),
$$

where $I_{k}(\mathcal{D})=\left\{I d_{k}(u) \mid u \in \mathcal{D}\right\}$.

Indeed, it is easy to see that $\operatorname{Id}(\mathcal{D})$ is an ideal for any domain $\mathcal{D}$. The converse construction is also possible.

Definition 14. For any ideal $I \subseteq 2^{A}$, we denote by Dom(I) the corresponding domain consisting of all linear orders that correspond to the flags contained in $I$.

The following lemma provides a missing link in our jigsaw puzzle.

\section{Lemma 1.}

(a) If $\mathcal{D} \subseteq \mathcal{L}(A)$ is a peak-pit domain of maximal width, then its ideal $\operatorname{Id}(\mathcal{D})$ is a separated ideal. 
(b) Conversely, if $S$ is a separated ideal, then the domain $\operatorname{Dom}(S)$ is a peak-pit domain of maximal width.

Proof. Part (a) was proved in Danilov et al. [2012]. To show part (b), we first note that $\mathcal{D}=\operatorname{Dom}(S)$ has maximal width by Proposition 2. We must prove that $\operatorname{Dom}(S)$ is a peak-pit domain. Suppose $\mathcal{D}=\operatorname{Dom}(S)$ is not a peak-pit domain. Then for some triple $\{a, b, c\}$ with $a<b<c$ the restriction $\mathcal{D}_{\{a, b, c\}}$ neither satisfies a never-top nor a never-bottom condition. However, due to maximal width $\mathcal{D}_{\{a, b, c\}} \supseteq\{a b c, c b a\}$. Then, there are four options for this restriction: $\{a b c, c b a, b a c, a c b\}$, or $\{a b c, c b a, b a c, c a b\}$, or $\{a b c, c b a, b c a, a c b\}$, or $\{a b c, c b a, c a b, b c a\}$, among which only the second and the third do not contain a cyclic triple. For these two remaining cases there will be two orders $x=x_{1} \ldots x_{n}$ and $y=y_{1} \ldots y_{n}$ from $\operatorname{Dom}(S)$ such that for some positive integers $k, m \in[n]$,

(i) $\operatorname{Id}_{k}(x) \cap\{a, b, c\}=\{b\}$, and

(ii) $\operatorname{Id}_{m}(y) \cap\{a, b, c\}=\{a, c\}$.

Let $S_{1}=\left\{x_{1}, \ldots, x_{k}\right\}$ and $S_{2}=\left\{y_{1}, \ldots, y_{m}\right\}$. Then $S_{1} \cap\{a, b, c\}=\{b\}$ and $S_{2} \cap\{a, b, c\}=$ $\{a, c\}$, which contradicts the fact that $S_{1}, S_{2} \in S$ are separated.

Example 1. Let $I=\bigcup_{i=0}^{4} I_{i} \subseteq 2^{[n]}$, where

$$
\begin{aligned}
& I_{0}=\{\emptyset\} \\
& I_{1}=\{\{1\},\{3\},\{4\}\} \\
& I_{2}=\{\{1,2\},\{1,3\},\{3,4\}\} \\
& I_{3}=\{\{1,2,3\},\{1,3,4\},\{2,3,4\}\} \\
& I_{4}=\{\{1,2,3,4\}\} .
\end{aligned}
$$

(Note that this ideal coincides with the collection of chamber sets of the arrangement in Figure 2.) The flags contained in I are

$$
\begin{aligned}
& 1 \rightarrow 12 \rightarrow 123 \rightarrow 1234 \\
& 1 \rightarrow 13 \rightarrow 132 \rightarrow 1324 \\
& 1 \rightarrow 13 \rightarrow 134 \rightarrow 1342 \\
& 3 \rightarrow 31 \rightarrow 312 \rightarrow 3124 \\
& 3 \rightarrow 31 \rightarrow 314 \rightarrow 3142
\end{aligned}
$$$$
3 \rightarrow 34 \rightarrow 341 \rightarrow 3412
$$$$
3 \rightarrow 34 \rightarrow 342 \rightarrow 3421
$$$$
4 \rightarrow 43 \rightarrow 431 \rightarrow 4312
$$$$
4 \rightarrow 43 \rightarrow 432 \rightarrow 4321
$$

and we can see that $\operatorname{Dom}(I)=F_{4}$.

For general domains we obtain the following result. 


\section{Proposition 6.}

(a) For each Condorcet domain $\mathcal{D}$ we have $\operatorname{Dom}(\operatorname{Id}(\mathcal{D})) \supseteq \mathcal{D}$.

(b) For each ideal $I \subseteq 2^{A}$ we have $\operatorname{Id}(\operatorname{Dom}(I)) \supseteq I$.

Proof. (a) If $u \in \mathcal{D}$, then it defines the following flag in $\operatorname{Id}(\mathcal{D})$ :

$$
\emptyset=\operatorname{Id}_{0}(u) \subset \operatorname{Id}_{1}(u) \subset \ldots \subset \operatorname{Id}_{n}(u),
$$

which will again give us $u \in \operatorname{Dom}(\operatorname{Id}(\mathcal{D}))$.

(b) Let $X \in I$. Then there is a flag $F=\left(F_{0}, F_{1}, \ldots, F_{n}\right)$ belonging to $I$ such that $X=F_{k}$ for some $i \in[n]$. This means that there is an order $u=a_{1}, \ldots, a_{n} \in \operatorname{Dom}(I)$ such that $X=\left\{a_{1}, \ldots, a_{k}\right\}$. But then $X=\operatorname{Id}_{k}(u)$ belongs to $\operatorname{Id}(\operatorname{Dom}(I))$. Hence $I \subseteq$ $\operatorname{Id}(\operatorname{Dom}(I))$.

However, we cannot expect to always have equality in (a) and (b) of Proposition 6 for arbitrary domains, even if these domains are maximal Condorcet domains. Here is an example.

Example 2. Let us consider for a counterexample the maximal never-middle Condorcet domain $\mathcal{D}=\{123,321,132,231\}$. Its ideal $I=\operatorname{Id}(\mathcal{D})$ is

$$
\begin{aligned}
& I_{0}=\{\emptyset\}, \\
& I_{1}=\{\{1\},\{2\},\{3\}\}, \\
& I_{2}=\{\{1,2\},\{1,3\},\{2,3\}\}, \\
& I_{3}=\{\{1,2,3\}\} .
\end{aligned}
$$

We see that $\operatorname{Dom}(I) \supsetneq \mathcal{D}$ as $\operatorname{Dom}(I)$ is the universal domain $\mathcal{L}([3])$. In particular, we see that $\operatorname{Id}(\mathcal{D})$ is not separated.

The following result shows that the equality $\operatorname{Dom}(\operatorname{Id}(\mathcal{D}))=\mathcal{D}$ holds for maximal peak-pit domains.

Theorem 2. (a) Let $\mathcal{D} \subseteq \mathcal{L}(A)$ be a Condorcet domain of maximal width. Then $\mathcal{D}$ is a peak-pit Condorcet domain if and only if its ideal $\operatorname{Id}(\mathcal{D})$ is separated. Moreover, if $\mathcal{D}$ is maximal, then

$$
\operatorname{Dom}(\operatorname{Id}(\mathcal{D}))=\mathcal{D} \text {. }
$$

(b) If $S$ is a maximal collection of separated subsets, then

$$
\operatorname{Id}(\operatorname{Dom}(S))=S \text {. }
$$

Proof. (a) If $\mathcal{D}$ is peak-pit, by Lemma 1 (a) we conclude that $\operatorname{Id}(\mathcal{D})$ is a separated ideal. And, if the ideal $\operatorname{Id}(\mathcal{D})$ is separated, then by part (b) of the same lemma we conclude that $\operatorname{Dom}(\operatorname{Id}(\mathcal{D}))$ is a peak-pit domain. By Proposition 6(a) the latter contains $\mathcal{D}$, hence $\mathcal{D}$ is a peak-pit domain. If $\mathcal{D}$ is maximal, then $\operatorname{Dom}(\operatorname{Id}(\mathcal{D}))=\mathcal{D}$ which proves (5).

(b) By Proposition $4 S$ is an ideal. Let $X \in S$. Since $S$ is an ideal, $X$ belongs to a certain flag and, hence $X$ will belong to $\operatorname{Id}(\operatorname{Dom}(S))$. Hence $S \subseteq \operatorname{Id}(\operatorname{Dom}(S))$. By Lemma $1(\operatorname{Dom}(S)$ is a peak-pit domain and $\operatorname{Id}(\operatorname{Dom}(S))$ is separated. But $S$ is maximal separated subset, hence $\operatorname{Id}(\operatorname{Dom}(S))=S$. 
Corollary 1. A domain $\mathcal{D}$ is a maximal Condorcet peak-pit domain of maximal width if and only if its ideal $\operatorname{Id}(\mathcal{D})$ is a maximal separated ideal.

Proof. Suppose $\operatorname{Id}(\mathcal{D})$ is not maximal and there is a larger separated ideal $I$ and $X \in$ $I \backslash \operatorname{Id}(\mathcal{D})$. Since $I$ is an ideal, there is a flag containing in $I$ that includes $X$. The linear order corresponding to this flag is not in $\operatorname{Id}(\mathcal{D})$.

If $\mathcal{D}$ is not maximal, then there is a larger peak-pit Condorcet domain $\mathcal{E} \supset \mathcal{D}$ and there is a linear order $u \in \mathcal{E} \backslash \mathcal{D}$. This means that there is a flag containing in $\operatorname{Id}(\mathcal{E})$ but not in $\operatorname{Id}(\mathcal{D})$. This means the latter is not maximal.

Proposition 7. Let $\mathcal{D}$ be a domain on the set of alternatives $A$ of cardinality $n$. Let $I=I d(\mathcal{D})=\bigcup_{k=0}^{n} I_{k}$ be the ideal of $\mathcal{D}$. Then $\bar{I}=\bigcup_{k=0}^{n} \bar{I}_{k}$, where $\bar{I}_{k}=\left\{[n] \backslash J \mid J \in I_{n-k}\right.$, is the ideal of the flipped domain $\overline{\mathcal{D}}$.

The proof is straightforward.

Example 3. We see that the flipped domain $\bar{F}_{4}$ of $F_{4}$ has the ideal (see Figure 3)

$$
\begin{aligned}
& \left(\bar{F}_{4}\right)_{0}=\{\emptyset\}, \\
& \left(\bar{F}_{4}\right)_{1}=\{\{1\},\{2\},\{4\}\}, \\
& \left(\bar{F}_{4}\right)_{2}=\{\{1,2\},\{2,4\},\{3,4\}\}, \\
& \left(\bar{F}_{4}\right)_{3}=\{\{1,2,3\},\{1,2,4\},\{2,3,4\}\}, \\
& \left(\bar{F}_{4}\right)_{4}=\{\{1,2,3,4\}\} .
\end{aligned}
$$

Hence this flipped $F_{4}$ is isomorphic to $F_{4}$ itself via $\sigma(i)=5-i$.

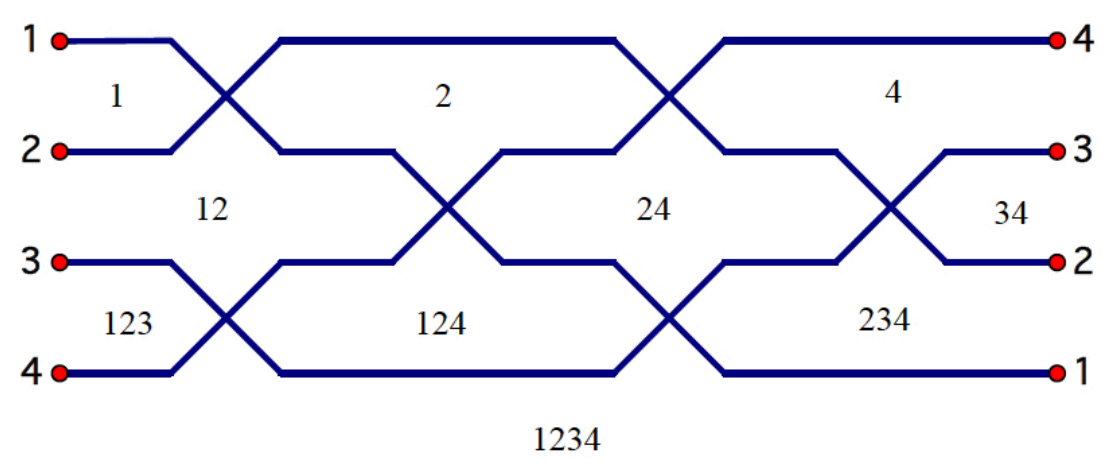

Figure 3: Arrangement of pseudolines for $\bar{F}_{4}$.

\subsection{Some important consequences}

Now that we know the equivalence of classes of equivalent reduced decompositions, arrangements of pseudolines, and Condorcet domains of maximal width, we can derive some important consequences. 
Theorem 3. Any maximal peak-pit domain $\mathcal{D} \subseteq \mathcal{L}([n])$ of maximal width is directly connected.

Proof. Firstly, we need to prove semi-connectedness. We know that $\mathcal{D}=\operatorname{Dom}(C(\mathbf{a}))$ for some arrangement of $n$ pseudolines a. Let $\left(i_{1}, \ldots, i_{p}\right)$ where $p=\frac{1}{2} n(n-1)$ be any reduced decomposition from the equivalence class of reduced decompositions corresponding to $\mathbf{a}$. Then as we know

$$
\bar{e}=e s_{i_{1}} \ldots s_{i_{p}},
$$

This means that $e$ can be transformed into $\bar{e}$ by performing transpositions $s_{i_{1}}, \ldots, s_{i_{p}}$ which means that $\mathcal{D}$ is semi-connected.

To prove the direct connectedness of $\mathcal{D}$, we need to use that the graph $G_{\mathcal{D}}$ is a median graph. By contradiction, assume that $\mathcal{D}$ is not directly connected; then, there exist two vertices $u, v \in G_{\mathcal{D}}$ such that $[u, v]_{\mathcal{D}}=\{u, v\}$ but $u$ and $v$ are not neighbors in the permutahedron. In such a case, for any third linear order $w \in \mathcal{D}$ the median $m=$ $m(w, u, v)$ must be equal to $u$ or to $v$ since it lies at the intersection of shortest paths from $u$ to $v$, from $u$ to $w$ and from $v$ to $w$.

Since $u$ and $v$ are not neighbours in the permutahedron we have two distinct pairs of alternatives $(a, b)$ and $(c, d)$ such that $a>_{u} b$ and $c>_{u} d$ but $b>_{v} a$ and $d>_{v} c$. Since $\mathcal{D}$ is semi-connected, there is a shortest path $P \subseteq \mathcal{D}$ that connects $e$ and $\bar{e}$. Then there must be a linear order $w$ on this path such that either $a>_{w} b$ and $d>_{w} c$ or $b>_{w} a$ and $c>{ }_{w} d$. Without loss of generality, suppose the former. Then the majority relation for $u, v, w$ must be $m \in\{u, v\}$. But we have $a>_{m} b$ and $d>_{m} c$. So $m \neq u$ and $m \neq v$, a contradiction.

The condition of maximality in Theorem 3 cannot be dropped as we can see from the following example.

Example 4. Let $A=\{a, b, c, d\}$ and

$$
\mathcal{N}=\left\{b N_{\{a, b, c\}} 1, b N_{\{a, b, d\}} 3, c N_{\{a, c, d\}} 1, c N_{\{b, c, d\}} 3\right\} .
$$

It is easily verified that $\mathcal{D}(\mathcal{N})=\{a b c d, d c b a\}$, which is a peak-pit domain with maximal width; evidently, it is not connected.

Corollary 2. Any maximal peak-pit Condorcet domain $\mathcal{D}$ of maximal width is copious.

Proof. Follows immediately from Theorem 3. Let $\{i, j, k\} \subseteq[n]$ such that $i<j<k$. Then the restriction $\mathcal{D}_{\{i, j, k\}}$ onto this subset will contain $i j k$ and $k j i$ (as $\mathcal{D}$ is of maximal width) and will be semi-connected, thus the restriction must contain at least two other linear orders.

Theorem 4. Let $\mathcal{D}$ be a maximal peak-pit Condorcet domain on $n$ alternatives of maximal width. Then

$$
|\operatorname{Id}(\mathcal{D})|=\left(\begin{array}{c}
n+1 \\
2
\end{array}\right)+1 .
$$


Proof. Since any two pseudolines cross exactly once, we have $\left(\begin{array}{c}n+1 \\ 2\end{array}\right)$ vertices in the corresponding arrangement of pseudolines. Each of them adds an additional chamber set.

In the next section, we will see that maximal peak-pit domains of maximal width can have quite different sizes. It is quite remarkable that the size of their ideals is constant.

\section{Classification of maximal peak-pit Condorcet do- mains of maximal width}

We formulate the following lemmas for the chamber sets of arrangements of pseudolines but due to Theorem 1 they are applicable to any maximal separated ideal.

Lemma 2. In each row of chamber sets of an arrangement of pseudolines the chambers are situated in the lexicographically increasing order from left to right.

Proof. Any two neighboring chambers have a common vertex in common. At this vertex for some $i<j$ lines $i$ and $j$ cross and an inversion happens, i.e., line $i$ from being above line $j$ goes under line $j$. Hence the chamber $X \cup\{i\}$ will have its right neighbor $X \cup\{j\}$ which is lexicographically larger.

Lemma 3. Let $C(\mathbf{a})=\bigcup_{i=0}^{n} I_{i}$ be an ideal of an arrangements of pseudolines $\mathbf{a}$. If for some $X \in I_{k-1}$ the sets $X \cup\{i\} \in I_{k}$ and $X \cup\{j\} \in I_{k}$, then

$$
X \cup\{i, j\} \in I_{k+1}
$$

if and only if $X \cup\{i\} \in I_{k}$ and $X \cup\{j\} \in I_{k}$ are neighbours in $I_{k}$.

Proof. If $X \cup\{i\}$ and $X \cup\{j\}$ are neighbours, this means lines $i$ and $j$ cross here making a vertex. Below this vertex we will have set $X \cup\{i, j\}$.

We will now demonstrate the power of the theory developed above by showing how to classify maximal peak-pit Condorcet domains of maximal width for $n=4$ and $n=5$.

\subsection{Classification of maximal peak-pit Condorcet domains of maximal width for $n=4$}

Due to Theorem 2 and Corollary 1, it is sufficient to classify maximal separated ideals in $2^{[4]}$. By Proposition 2 such an ideal will always contain sets

$$
\emptyset,\{1\},\{1,2\},\{1,2,3\},\{1,2,3,4\},\{2,3,4\},\{3,4\},\{4\} .
$$

By Theorem 4 the cardinality of a maximal separated ideal is 11 , so only three other sets can be added to (7). Some choices may preclude some other ones. Let us list incompatible pairs of sets (omitting curly brackets around sets):

$$
\begin{aligned}
& (2,13),(2,14),(2,134),(3,14),(3,24),(3,124), \\
& (13,24),(23,14),(24,134),(23,134),(23,124) .
\end{aligned}
$$

We have the following choices: 
- The ideal has four singletons. $I_{1}=\{\{1\},\{2\},\{3\},\{4\}\}$. In this case we can add only $\{2,3\}$ in $I_{2}$ and after inclusion of it $I$ becomes maximal. Thus, the ideal is

$$
\begin{aligned}
& I_{0}=\{\emptyset\}, \\
& I_{1}=\{\{1\},\{2\},\{3\},\{4\}\}, \\
& I_{2}=\{\{1,2\},\{2,3\},\{3,4\}\}, \\
& I_{3}=\{\{1,2,3\},\{2,3,4\}\}, \\
& I_{4}=\{\{1,2,3,4\}\} .
\end{aligned}
$$

This is the ideal of a maximal single-peaked domain on four alternatives:

$$
S P_{4}=\{1234,2134,2314,3214,2341,3241,3421,4321\},
$$

whose median graph is presented on Figure 4.

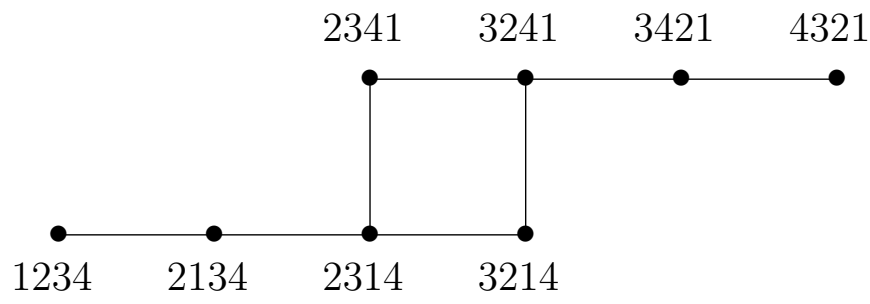

Figure 4: Graph of the single-peaked domain $\mathrm{SP}_{4}$ on four alternatives

Since no sets can be added to $I_{3}$ this is the only domain with four singletons in $I_{1}{ }^{6}$

- The ideal has three singletons. Because of the isomorphism $\sigma(i)=5-i$, we may assume without loss of generality that $I_{1}=\{\{1\},\{2\},\{4\}\}$. As $\{2\}$ belongs to the ideal, at level 2 we cannot have $\{1,3\}$ or $\{1,4\}$, nor $\{1,3,4\}$ at level 3 . However, by Lemma 3 we must include $\{1,2\},\{2,4\},\{3,4\}$; for the remaining 11th set we are left with two choices:

(a) include $\{2,3\}$ or

(b) include $\{1,2,4\}$.

The case (b), as we saw, leads to Fishburn's domain $\bar{F}_{4}$ and in case (a) we have the

\footnotetext{
${ }^{6}$ Having all singletons present means that the domain is minimally rich in the sense of Puppe [2018] who showed that the single-peaked domain is in fact the only semi-connected Condorcet domain with that property.
} 
ideal

$$
\begin{aligned}
& I_{0}=\{\emptyset\} \\
& I_{1}=\{\{1\},\{2\},\{4\}\} \\
& I_{2}=\{\{1,2\},\{2,3\},\{2,4\},\{3,4\}\} \\
& I_{3}=\{\{1,2,3\},\{2,3,4\}\} \\
& I_{4}=\{\{1,2,3,4\}\} .
\end{aligned}
$$

which gives us the single-crossing domain

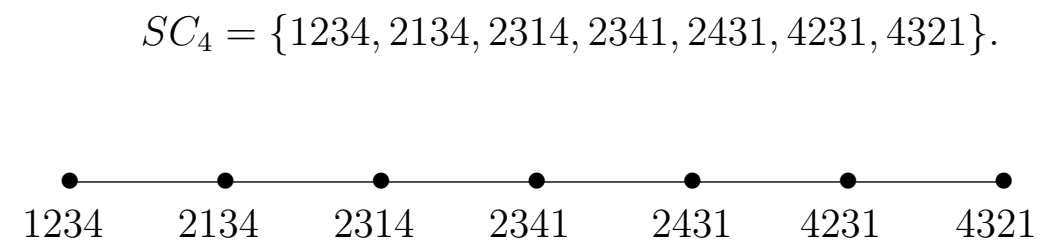

Figure 5: Graph of single-crossing domain $S C_{4}$ on four alternatives

- The ideal has two singletons. We claim that up to a flip-isomorphism this case has already been dealt with. Indeed, the flip-isomorphic domain has as many singletons as the number of sets in $I_{3}$. If this level also has two sets, then by Theorem 4 we should have five sets out of six possible at level 2. However, the sets in pairs $(13,24),(23,14)$ are incompatible, hence this case is impossible.

Theorem 5. Up to an isomorphism and flip-isomorphism there exist only three maximal peak-pit Condorcet domains of maximal width: the single-crossing, the single-peaked and the Fishburn domains.

\subsection{Classification of maximal peak-pit Condorcet domains of maximal width for $n=5$}

By our preceding analysis, we know that the ideal of any maximal peak-pit domain of maximal width on a set of $n=5$ alternatives has cardinality 16 and will include the following 10 sets:

$$
\emptyset,\{1\},\{1,2\},\{1,2,3\},\{1,2,3,4\},\{1,2,3,4,5\},\{2,3,4,5\},\{3,4,5\},\{4,5\},\{5\} .
$$


A singleton $\{i\}$ is not compatible with any set containing $\{j, k\}$ with $j<i<k$ but not $i$. Also, the following pairs and triples of alternatives are incompatible:

$(1,3)$ is incompatible with $(2,4),(2,5)$ and $(2,4,5)$;

$(1,4)$ is incompatible with $(2,5),(3,5)$ and $(2,3,5)$;

$(1,5)$ is incompatible with $(2,3),(3,4)$, and $(2,4),(2,3,4)$;

$(2,4)$ is incompatible with $(1,3)$ and $(3,5)$ and $(1,3,5)$;

$(2,5)$ is incompatible with $(1,3),(1,4)$ and $(3,4),(1,3,4)$;

$(3,5)$ is incompatible with $(1,4)$ and $(2,4)$ and $(1,2,4)$.

The following observation will simplify the classification.

Lemma 4. Let $\mathcal{D}$ be a maximal peak-pit domain of maximal width over five alternatives such that its ideal $I(\mathcal{D})=\bigcup_{i=0}^{5} I_{i}$ contains exactly two singletons in $I_{1}$. Then $I_{4}$ must contains at least three sets.

Proof. Suppose the ideal of $\mathcal{D}$ has two singletons in $I_{1}$. Then $I_{1}=\{\{1\},\{5\}\}$ and by Lemma $3, I_{2}$ contains the set $\{1,5\}$. Hence there must be a flag containing $\{1,5\}$. The set of cardinality four from this flag belongs to $I_{4}$ and it is different from $\{1,2,3,4\}$ and $\{2,3,4,5\}$ which are always in $I_{4}$ due to maximal width. Hence $I_{4}$ contains at least three sets.

Corollary 3. Any peak-pit Condorcet domain of maximal width is isomorphic or flipisomorphic to a peak-pit domain of maximal width whose ideal contains at least three singletons.

Proof. By Lemma 4 and Proposition 7 if a domain has two singletons, then its flipped domain contains at least three.

I. Five singletons. Suppose we have a maximal ideal $I$ such that $I_{1}$ contains all five singletons.

Domain 1. By Lemma 3 all other options are forced and we cannot add anything anywhere:

$$
\begin{aligned}
& I_{0}=\{\emptyset\}, \\
& I_{1}=\{\{1\},\{2\},\{3\},\{4\},\{5\}\}, \\
& I_{2}=\{\{1,2\},\{2,3\},\{3,4\},\{4,5\}\}, \\
& I_{3}=\{\{1,2,3\},\{2,3,4\},\{3,4,5\}\}, \\
& I_{4}=\{\{1,2,3,4\},\{2,3,4,5\}\}, \\
& I_{5}=\{\{1,2,3,4,5\}\} .
\end{aligned}
$$


This is the single-peaked domain. It contains 16 linear orders and is characterized by zero 'inversion triples' (cf. Galambos and Reiner [2008]).

II. Four singletons. Suppose we have a maximal ideal $I$ such that $I_{1}$ contains four singletons. Then, up to an isomorphism, we have either $I_{1}=\{\{1\},\{2\},\{3\},\{5\}\}$ or $I_{1}=\{\{1\},\{2\},\{4\},\{5\}\}$.

Domain 2. Let $I_{1}=\{\{1\},\{2\},\{3\},\{5\}\}$. Lemma 3 forces us to choosing $\{2,3\},\{3,5\}$ to $I_{2}$. If no other sets are added to $I_{2}$, we must, again by Lemma 3 , add $\{2,3,5\}$ to $I_{3}$ and $\{1,2,3,5\}$ to $I_{4}$. The resulting ideal is maximal since it contains 16 sets:

$$
\begin{aligned}
& I_{0}=\{\emptyset\}, \\
& I_{1}=\{\{1\},\{2\},\{3\},\{5\}\}, \\
& I_{2}=\{\{1,2\},\{2,3\},\{3,5\},\{4,5\}\}, \\
& I_{3}=\{\{1,2,3\},\{2,3,5\},\{3,4,5\}\}, \\
& I_{4}=\{\{1,2,3,4\},\{1,2,3,5\},\{2,3,4,5\}\}, \\
& I_{5}=\{\{1,2,3,4,5\}\} .
\end{aligned}
$$

The corresponding domain contains 19 linear orders and is defined by the following inversion triples:

$$
[1,4,5],[2,4,5],[3,4,5] \text {. }
$$

Domain 3. $I_{1}=\{\{1\},\{2\},\{3\},\{5\}\}$. Unlike with Domain 2, we can add $\{3,4\}$ at level 2 which will force us to replace $\{2,3,5\}$ with $\{2,3,4\}$ and will preclude $\{1,2,3,5\}$ at level 4:

$$
\begin{aligned}
& I_{0}=\{\emptyset\}, \\
& I_{1}=\{\{1\},\{2\},\{3\},\{5\}\}, \\
& I_{2}=\{\{1,2\},\{2,3\},\{3,4\},\{3,5\},\{4,5\}\}, \\
& I_{3}=\{\{1,2,3\},\{2,3,4\},\{3,4,5\}\}, \\
& I_{4}=\{\{1,2,3,4\},\{2,3,4,5\}\}, \\
& I_{5}=\{\{1,2,3,4,5\}\} .
\end{aligned}
$$

This ideal generates a domain containing 14 linear orders which is defined by a single inversion triple:

$$
[3,4,5] \text {. }
$$

Domain 4. $I_{1}=\{\{1\},\{2\},\{3\},\{5\}\}$. Also, instead of $\{3,4\}$, as in Domain 3, we can add $\{2,3,4\}$ and $\{2,3,5\}$ to $I_{3}$. This also precludes $\{1,2,3,5\}$ at level 4 . Then,

$$
\begin{aligned}
& I_{0}=\{\emptyset\}, \\
& I_{1}=\{\{1\},\{2\},\{3\},\{5\}\}, \\
& I_{2}=\{\{1,2\},\{2,3\},\{3,5\},\{4,5\}\}, \\
& I_{3}=\{\{1,2,3\},\{2,3,4\},\{2,3,5\},\{3,4,5\}\}, \\
& I_{4}=\{\{1,2,3,4\},\{2,3,4,5\}\}, \\
& I_{5}=\{\{1,2,3,4,5\}\} .
\end{aligned}
$$


This domain contains 15 linear orders and is defined by the inversion triples:

$$
[2,4,5],[3,4,5] \text {. }
$$

Domain 5. Now let $I_{1}=\{\{1\},\{2\},\{4\},\{5\}\}$. This and Lemma 3 forces us to choosing $\{2,4\}$ to $I_{2},\{1,2,4\}$ and $\{2,4,5\}$ to $I_{3}$, and $\{1,2,4,5\}$ to $I_{4}$. The resulting ideal is already maximal since it contains 16 sets.

$$
\begin{aligned}
& I_{0}=\{\emptyset\}, \\
& I_{1}=\{\{1\},\{2\},\{4\},\{5\}\}, \\
& I_{2}=\{\{1,2\},\{2,4\},\{4,5\}\}, \\
& I_{3}=\{\{1,2,3\},\{1,2,4\},\{2,4,5\},\{3,4,5\}\}, \\
& I_{4}=\{\{1,2,3,4\},\{1,2,4,5\},\{2,3,4,5\}\}, \\
& I_{5}=\{\{1,2,3,4,5\}\} .
\end{aligned}
$$

This is one of two Fishburn's domains. It contains 20 linear orders and is defined by the inversion triples:

$$
[1,3,4],[1,3,5],[2,3,4],[2,3,5] \text {. }
$$

With the same level 1 , we can either add $\{2,3\}$ and/or $\{3,4\}$ to $I_{2}$, and/or add $\{2,3,4\}$ to $I_{3}$. We note that adding $\{3,4\}$ will give us a domain isomorphic to that when we add $\{2,3\}$.

Domain 6. Let $I_{1}=\{\{1\},\{2\},\{4\},\{5\}\}$ and adding $\{2,3\}$ to $I_{2}$ in addition to those that are forced by Lemma 3. Then

$$
\begin{aligned}
& I_{0}=\{\emptyset\}, \\
& I_{1}=\{\{1\},\{2\},\{4\},\{5\}\}, \\
& I_{2}=\{\{1,2\},\{2,3\},\{2,4\},\{4,5\}\}, \\
& I_{3}=\{\{1,2,3\},\{2,3,4\},\{2,4,5\},\{3,4,5\}\}, \\
& I_{4}=\{\{1,2,3,4\},\{2,3,4,5\}\}, \\
& I_{5}=\{\{1,2,3,4,5\}\} .
\end{aligned}
$$

This domain contains 15 linear orders defined by the inversion triples:

$$
[2,3,4],[2,3,5] \text {. }
$$

Domain 7. Let $I_{1}=\{\{1\},\{2\},\{4\},\{5\}\}$ and adding both $\{2,3\}$ and $\{3,4\}$ to $I_{2}$ : Then

$$
\begin{aligned}
& I_{0}=\{\emptyset\}, \\
& I_{1}=\{\{1\},\{2\},\{4\},\{5\}\}, \\
& I_{2}=\{\{1,2\},\{2,3\},\{2,4\},\{3,4\},\{4,5\}\}, \\
& I_{3}=\{\{1,2,3\},\{2,3,4\},\{3,4,5\}\}, \\
& I_{4}=\{\{1,2,3,4\},\{2,3,4,5\}\}, \\
& I_{5}=\{\{1,2,3,4,5\}\} .
\end{aligned}
$$


This domain contains 14 linear orders and is defined by a single inversion triple:

$$
[2,3,4] \text {. }
$$

Domain 8. Let $I_{1}=\{\{1\},\{2\},\{4\},\{5\}\}$ and adding $\{2,3,4\}$ to $I_{3}$. Then

$$
\begin{aligned}
& I_{0}=\{\emptyset\}, \\
& I_{1}=\{\{1\},\{2\},\{4\},\{5\}\}, \\
& I_{2}=\{\{1,2\},\{2,4\},\{4,5\}\}, \\
& I_{3}=\{\{1,2,3\},\{1,2,4\},\{2,3,4\},\{2,4,5\},\{3,4,5\}\}, \\
& I_{4}=\{\{1,2,3,4\},\{2,3,4,5\}\}, \\
& I_{5}=\{\{1,2,3,4,5\}\} .
\end{aligned}
$$

This domain contains 16 linear orders defined by the inversion triples:

$$
[1,3,4],[2,3,4],[2,3,5] \text {. }
$$

It will be useful to have the following statement for future reference.

Lemma 5. If an ideal I has $\left|I_{4}\right| \geq 4$, then the corresponding domain is flip-isomorphic to one of the domains 2-8.

Proof. Follows from Proposition 7.

III. Three singletons. Suppose we have a maximal ideal $I$ such that $I_{1}$ contains three singletons. Then, up to an isomorphism, we have either $I_{1}=\{\{1\},\{2\},\{5\}\}$ or $I_{1}=$ $\{\{1\},\{3\},\{5\}\}$. Let us consider the first case.

Let $I_{1}=\{\{1\},\{2\},\{5\}\}$ and $I_{2}=\{\{1,2\},\{2,5\},\{4,5\}\}$ which is the minimum required by Lemma 3. Then, if we do not add anything to $I_{2}$ and $I_{3}$, then it is easy to see that we will have four elements in $I_{4}$ and thus this case is flip-isomorphic to cases considered already by Lemma 5 .

Let us start with additions to level 2 . The pairs that are compatible with $I_{1}$ and may be included are:

$$
\{2,3\},\{2,4\},\{3,5\} \text {. }
$$

However we note that $\{2,4\}$ and $\{3,5\}$ are mutually incompatible.

Domain 9. Let $I_{1}=\{\{1\},\{2\},\{5\}\}$ and we add only $\{2,3\}$. Then everything is determined:

$$
\begin{aligned}
& I_{0}=\{\emptyset\}, \\
& I_{1}=\{\{1\},\{2\},\{5\}\}, \\
& I_{2}=\{\{1,2\},\{2,3\},\{2,5\},\{4,5\}\}, \\
& I_{3}=\{\{1,2,3\},\{2,3,5\},\{2,4,5\},\{3,4,5\}\}, \\
& I_{4}=\{\{1,2,3,4\},\{1,2,3,5\},\{2,3,4,5\}\}, \\
& I_{5}=\{\{1,2,3,4,5\}\} .
\end{aligned}
$$


This domain contains 16 linear orders and is defined by the inversion triples:

$$
[1,4,5],[2,3,4],[2,3,5],[2,4,5],[3,4,5] \text {. }
$$

Domain 10. Let $I_{1}=\{\{1\},\{2\},\{5\}\}$ and we add only $\{2,4\}$. Then by Lemma 3 everything else is determined:

$$
\begin{aligned}
& I_{0}=\{\emptyset\}, \\
& I_{1}=\{\{1\},\{2\},\{5\}\}, \\
& I_{2}=\{\{1,2\},\{2,4\},\{2,5\},\{4,5\}\}, \\
& I_{3}=\{\{1,2,3\},\{1,2,4\},\{2,4,5\},\{3,4,5\}\}, \\
& I_{4}=\{\{1,2,3,4\},\{1,2,4,5\},\{2,3,4,5\}\}, \\
& I_{5}=\{\{1,2,3,4,5\}\} .
\end{aligned}
$$

This domain contains 17 linear orders and is defined by the inversion triples:

$$
[1,3,4],[1,3,5],[2,3,4],[2,3,5],[2,4,5] \text {. }
$$

Domain 11. Let $I_{1}=\{\{1\},\{2\},\{5\}\}$ and we add both $\{2,3\}$ and $\{2,4\}$. Then by Lemma 3 everything is determined:

$$
\begin{aligned}
& I_{0}=\{\emptyset\}, \\
& I_{1}=\{\{1\},\{2\},\{5\}\}, \\
& I_{2}=\{\{1,2\},\{2,3\},\{2,4\},\{2,5\},\{4,5\}\}, \\
& I_{3}=\{\{1,2,3\},\{2,3,4\},\{2,4,5\},\{3,4,5\}\}, \\
& I_{4}=\{\{1,2,3,4\},\{2,3,4,5\}\}, \\
& I_{5}=\{\{1,2,3,4,5\}\} .
\end{aligned}
$$

This domain contains 12 linear orders and is defined by the inversion triples:

$$
[2,3,4],[2,3,5],[2,4,5] \text {. }
$$

Let $I_{1}=\{\{1\},\{2\},\{5\}\}$ and we add only $\{3,5\}$. Then everything is determined:

$$
\begin{aligned}
& I_{0}=\{\emptyset\}, \\
& I_{1}=\{\{1\},\{2\},\{5\}\}, \\
& I_{2}=\{\{1,2\},\{2,5\},\{3,5\},\{4,5\}\}, \\
& I_{3}=\{\{1,2,3\},\{1,2,5\},\{2,3,5\},\{3,4,5\}\}, \\
& I_{4}=\{\{1,2,3,4\},\{1,2,3,5\},\{2,3,4,5\}\}, \\
& I_{5}=\{\{1,2,3,4,5\}\} .
\end{aligned}
$$

This domain is flip-isomorphic to Domain 9. 
Domain 12. Let $I_{1}=\{\{1\},\{2\},\{5\}\}$ and we add both $\{2,3\}$ and $\{3,5\}$. Then:

$$
\begin{aligned}
& I_{0}=\{\emptyset\}, \\
& I_{1}=\{\{1\},\{2\},\{5\}\}, \\
& I_{2}=\{\{1,2\},\{2,3\},\{2,5\},\{3,5\},\{4,5\}\}, \\
& I_{3}=\{\{1,2,3\},\{2,3,5\},\{3,4,5\}\}, \\
& I_{4}=\{\{1,2,3,4\},\{1,2,3,5\},\{2,3,4,5\}\}, \\
& I_{5}=\{\{1,2,3,4,5\}\} .
\end{aligned}
$$

This domain contains 16 linear orders and is defined by the inversion triples:

$$
[1,4,5],[2,3,5],[2,4,5],[3,4,5] \text {. }
$$

Now let us start adding triples as well. The triples that are compatible are $\{1,2,4\}$, $\{2,3,4\},\{2,3,5\}$. We note that $\{1,2,4\}$ is incompatible with $\{2,3\}$ and $\{3,5\}$ and we have already added it with $\{2,4\}$. If we add it alone, then

$$
\begin{aligned}
& I_{0}=\{\emptyset\}, \\
& I_{1}=\{\{1\},\{2\},\{5\}\}, \\
& I_{2}=\{\{1,2\},\{2,5\},\{4,5\}\}, \\
& I_{3}=\{\{1,2,3\},\{1,2,4\},\{1,2,5\},\{2,4,5\},\{3,4,5\}\}, \\
& I_{4}=\{\{1,2,3,4\},\{1,2,4,5\},\{2,3,4,5\}\}, \\
& I_{5}=\{\{1,2,3,4,5\}\} .
\end{aligned}
$$

From $I_{4}$ we observe that this domain is flip-isomorphic to the one whose ideal has $I_{1}=$ $\{\{1\},\{3\},\{5\}\}$. It will be flip-isomorphic to Domain 16 below.

We can also add $\{2,3,4\}$ but not alone as it is incompatible with $\{1,5\}$.

Domain 13. Let $I_{1}=\{\{1\},\{2\},\{5\}\}$ and we add both $\{2,3\}$ and $\{2,3,4\}$. Then

$$
\begin{aligned}
& I_{0}=\{\emptyset\}, \\
& I_{1}=\{\{1\},\{2\},\{5\}\}, \\
& I_{2}=\{\{1,2\},\{2,3\},\{2,5\},\{4,5\}\}, \\
& I_{3}=\{\{1,2,3\},\{2,3,4\},\{2,3,5\},\{2,4,5\},\{3,4,5\}\}, \\
& I_{4}=\{\{1,2,3,4\},\{2,3,4,5\}\}, \\
& I_{5}=\{\{1,2,3,4,5\}\} .
\end{aligned}
$$

This domain contains 12 linear orders and is defined by the inversion triples:

$$
[2,3,4],[2,3,5],[2,4,5],[3,4,5] \text {. }
$$


Domain 14. Let $I_{1}=\{\{1\},\{2\},\{5\}\}$ and we add both $\{2,4\},\{2,3,4\}$. Then

$$
\begin{aligned}
& I_{0}=\{\emptyset\}, \\
& I_{1}=\{\{1\},\{2\},\{5\}\}, \\
& I_{2}=\{\{1,2\},\{2,4\},\{2,5\},\{4,5\}\}, \\
& I_{3}=\{\{1,2,3\},\{1,2,4\},\{2,3,4\},\{2,4,5\},\{3,4,5\}\}, \\
& I_{4}=\{\{1,2,3,4\},\{2,3,4,5\}\}, \\
& I_{5}=\{\{1,2,3,4,5\}\} .
\end{aligned}
$$

This domain contains 12 linear orders and is defined by the inversion triples:

$$
[1,3,4],[2,3,4],[2,3,5],[2,4,5] \text {. }
$$

Domain 15. Let $I_{1}=\{\{1\},\{2\},\{5\}\}$ and we add $\{2,3\},\{3,5\}$ and $\{2,3,4\}$. Then

$$
\begin{aligned}
& I_{0}=\{\emptyset\}, \\
& I_{1}=\{\{1\},\{2\},\{5\}\}, \\
& I_{2}=\{\{1,2\},\{2,3\},\{2,5\},\{3,5\},\{4,5\}\}, \\
& I_{3}=\{\{1,2,3\},\{2,3,4\},\{2,3,5\},\{3,4,5\}\}, \\
& I_{4}=\{\{1,2,3,4\},\{2,3,4,5\}\}, \\
& I_{5}=\{\{1,2,3,4,5\}\} .
\end{aligned}
$$

It contains 11 linear orders and is defined by the inversion triples:

$$
[2,3,5],[2,4,5],[3,4,5] \text {. }
$$

This is a single-crossing domain.

As for $\{2,3,5\}$, we added it with $\{2,3\}$ and $\{3,5\}$ and it is not compatible with $\{2,4\}$. We can add it alone but this will be flip-isomorphic to Domain 12 .

Alternatively, we can start with $I_{1}=\{\{1\},\{3\},\{5\}\}$. We will then have to select $\{1,3\},\{3,5\}$ into $I_{2}$ due to Lemma 3 . If we add nothing else, then:

$$
\begin{aligned}
& I_{0}=\{\emptyset\}, \\
& I_{1}=\{\{1\},\{3\},\{5\}\}, \\
& I_{2}=\{\{1,2\},\{1,3\},\{3,5\},\{4,5\}\}, \\
& I_{3}=\{\{1,2,3\},\{1,3,5\},\{3,4,5\}\}, \\
& I_{4}=\{\{1,2,3,4\},\{1,2,3,5\},\{1,3,4,5\},\{2,3,4,5\}\}, \\
& I_{5}=\{\{1,2,3,4,5\}\} .
\end{aligned}
$$

This is one of Fishburn's domains, it is flip-isomorphic to Domain 5.

We can then add pairs or triples. Only the pairs $\{2,3\},\{3,4\}$, and the triples $\{2,3,4\}$, $\{1,3,4\},\{2,3,5\}$ are compatible to $\{1,3\},\{3,5\}$. However, $\{1,3,4\}$ and $\{2,3\}$ and also $\{2,3,5\}$ are incompatible. 
Domain 16. Let $I_{1}=\{\{1\},\{3\},\{5\}\}$ and we add $\{2,3\}$ to $I_{2}$ and nothing else This would be isomorphic to adding $\{3,4\}$. Then:

$$
\begin{aligned}
& I_{0}=\{\emptyset\}, \\
& I_{1}=\{\{1\},\{3\},\{5\}\}, \\
& I_{2}=\{\{1,2\},\{1,3\},\{2,3\},\{3,5\},\{4,5\}\}, \\
& I_{3}=\{\{1,2,3\},\{2,3,5\},\{3,4,5\}\}, \\
& I_{4}=\{\{1,2,3,4\},\{1,2,3,5\},\{2,3,4,5\}\}, \\
& I_{5}=\{\{1,2,3,4,5\}\} .
\end{aligned}
$$

This domain contains 17 linear orders and is defined by the inversion triples:

$$
[1,2,3], \quad[1,4,5], \quad[2,4,5], \quad[3,4,5] .
$$

Domain 17. Let $I_{1}=\{\{1\},\{3\},\{5\}\}$ and we add $\{2,3\}$ and $\{3,4\}$. Then:

$$
\begin{aligned}
& I_{0}=\{\emptyset\}, \\
& I_{1}=\{\{1\},\{3\},\{5\}\}, \\
& I_{2}=\{\{1,2\},\{1,3\},\{2,3\},\{3,4\},\{3,5\},\{4,5\}\}, \\
& I_{3}=\{\{1,2,3\},\{2,3,4\},\{3,4,5\}\}, \\
& I_{4}=\{\{1,2,3,4\},\{2,3,4,5\}\}, \\
& I_{5}=\{\{1,2,3,4,5\}\} .
\end{aligned}
$$

This domain contains 12 linear orders and is defined by the inversion triples:

$$
[1,2,3], \quad[3,4,5] \text {. }
$$

Domain 18. Let $I_{1}=\{\{1\},\{3\},\{5\}\}$ and we add $\{2,3\}$ and $\{2,3,4\}$, which would be isomorphic to adding $\{3,4\}$ and $\{2,3,4\}$. Then:

$$
\begin{aligned}
& I_{0}=\{\emptyset\}, \\
& I_{1}=\{\{1\},\{3\},\{5\}\}, \\
& I_{2}=\{\{1,2\},\{1,3\},\{2,3\},\{3,5\},\{4,5\}\} \\
& I_{3}=\{\{1,2,3\},\{2,3,4\},\{2,3,5\},\{3,4,5\}\}, \\
& I_{4}=\{\{1,2,3,4\},\{2,3,4,5\}\}, \\
& I_{5}=\{\{1,2,3,4,5\}\} .
\end{aligned}
$$

This domain contains 12 linear orders with the inversion triple:

$$
[1,2,3],[2,4,5],[3,4,5]
$$


then

We are left with the case when $I_{1}=\{\{1\},\{3\},\{5\}\}$ and we are adding $\{1,3,4\}$ to $I_{2}$,

$$
\begin{aligned}
& I_{0}=\{\emptyset\}, \\
& I_{1}=\{\{1\},\{3\},\{5\}\}, \\
& I_{2}=\{\{1,2\},\{1,3\},\{3,5\},\{4,5\}\}, \\
& I_{3}=\{\{1,2,3\},\{1,3,4\},\{1,3,5\},\{3,4,5\}\}, \\
& I_{4}=\{\{1,2,3,4\},\{1,3,4,5\},\{2,3,4,5\}\}, \\
& I_{5}=\{\{1,2,3,4,5\}\} .
\end{aligned}
$$

This domain is flip-isomorphic to Domain 10.

IV. Two singletons. This case is covered by Lemma 4 . In such a case $I_{4}$ must contains at least 3 sets and the corresponding domains are filp-isomorphic to one of the domains above.

Theorem 6. Up to isomorphism and flip-isomorphism, there exist exactly 18 maximal Condorcet peak-pit domains of maximal width on a set of $n=5$ alternatives.

The number of domains of different sizes is given in the following table:

\begin{tabular}{|c|c|c|c|c|c|c|c|c|c|c|}
\hline Size & 11 & 12 & 13 & 14 & 15 & 16 & 17 & 18 & 19 & 20 \\
\hline The number of domains & 1 & 5 & 0 & 2 & 2 & 4 & 2 & 0 & 1 & 1 \\
\hline
\end{tabular}

\section{Outlook and further research}

In the present paper, we have shown how the notion of an ideal of a domain can be used for the classification of peak-pit domains of maximal width. Remarkably, the calculations in Dittrich [2018] reveal that for $n=4$ all peak-pit Condorcet domains have ideals of cardinality 11 and are directly connected regardless of whether or not they have maximal width; for instance, the Arrow single-peaked domains identified in Slinko [2019] do not necessarily have maximal width but still possess these properties. It thus seems worthwhile to investigate to what extent the results of this paper hold for arbitrary maximal peak-pit Condorcet domains.

\section{References}

F. Aleskerov. Arrovian Aggregation Models. Kluwer, Dordrecht, 1999.

A. Björner, M. Las Vergnas, B. Sturmfels, N. White, and G.M. Ziegler. Oriented matroids. Cambridge University Press, 1999. 
V.I. Danilov and G.A. Koshevoy. Maximal Condorcet domains. Order, 30(1):181-194, 2013.

V.I. Danilov, A.V. Karzanov, and G.A. Koshevoy. Condorcet domains of tiling type. Discrete Applied Mathematics, 160(7-8):933-940, 2012.

T. Dittrich. Eine vollständige Klassifikation von Condorcet Domains für kleine Alternativenmengen. PhD thesis, Karlsruhe Institute of Technology, 2018.

S. Elnitsky. Rhombic tilings of polygons and classes of reduced words in coxeter groups. journal of combinatorial theory, Series A, 77(2):193-221, 1997.

P.C. Fishburn. Acyclic sets of linear orders. Social Choice and Welfare, 14(1):113-124, 1996.

A. Galambos and V. Reiner. Acyclic sets of linear orders via the Bruhat orders. Social Choice and Welfare, 30(2):245-264, 2008.

B. Grunbaum. Arrangements and spreads. In Conference Board of the Mathematical Sciences Regional Conference Series in Mathematics, volume 10. American Mathematical Society, 1972.

J. E. Humphreys. Reflection Groups and Coxeter Groups. Cambridge Univ. Press, Cambridge, 1994.

J. Kemeny. Mathematics without numbers. Daedalus, 88:577-591, 1959.

J. Kemeny and L. Snell. Mathematical Models in the Social Sciences. Ginn, 1960.

B. Leclerc and A. Zelevinsky. Quasicommuting families of quantum Plücker coordinates. In Kirillov's seminar on representation theory, volume 35, page 85, 1998.

B. Monjardet. Acyclic domains of linear orders: A survey. In S. Brams, W. Gehrlein, and F. Roberts, editors, The Mathematics of Preference, Choice and Order, Studies in Choice and Welfare, pages 139-160. Springer Berlin Heidelberg, 2009.

H. M. Mulder. The structure of median graphs. Discrete Math., 24:197-204, 1978.

C. Puppe. The single-peaked domain revisited: A simple global characterization. Working Paper 97, Karlsruhe Institute of Technology, 2016.

C. Puppe. The single-peaked domain revisited: A simple global characterization. Journal of Economic Theory, 176:55 - 80, 2018.

C. Puppe and A. Slinko. Condorcet domains, median graphs and the single-crossing property. Economic Theory, 67(1):285-318, 2019.

A. K. Sen. A Possibility theorem on majority decisions. Econometrica, 34(2):491-499, 1966. 
A. Slinko. Condorcet domains satisfying Arrow's single-peakedness. Journal of Mathematical Economics, 84:166-175, 2019.

\section{Appendix}

Here is the list of the linear orders in each of the 18 maximal peak-pit domains of maximal width for $n=5$ :

Domain 1 : 12345, 21345, 23145, 23415, 23451,32145, 32415, 32451,34215, 34251, $34521,43215,43251,43521,45321,54321$;

Domain 2 : 12345, 21345, 23145, 32145, 35421, 53421, 54321, 23514, 32514, 35214, $53214,23541,32541,35241,53241,12354,21354,23154,32154$;

Domain 3 : 12345, 21345, 23145, 32145, 35421, 53421, 54321, 23415, 23451,32415, $32451,34215,34251,34521$;

Domain 4 : 12345, 21345, 23145, 32145,35421, 53421, 54321, 23415, 23451,32415, $32451,23541,32541,35241,53241$;

Domain 5 : 12345, 12435, 12453, 21345, 21435, 21453, 24135, 24153, 24513, 24531, $42153,42135,42513,42531,45213,45231,45321,54213,54231,54321$;

Domain 6 : 12345, 21345, 23145, 23415, 23451, 24315, 24351, 42315, 42351, 45321, $54321,24531,42531,45231,54231$;

Domain 7 : 12345, 21345, 23145, 23415, 23451, 24315, 24351, 42315, 42351,45321, $54321,43215,43251,43521$;

Domain 8 : 12345, 12435, 21345, 21435, 24135, 24531, 42135, 42531, 45231, 45321, $54231,54321,24315,24351,42315,42351$;

Domain 9 : 12345, 21345, 23145, 25431, 52431, 54231, 54321, 23541, 25341, 52341, $12354,21354,23154,23514,25314,52314$;

Domain 10 : 12345, 12435, 21345, 21435, 25431, 52431, 54231, 54321, 12453, 21453, $25413,52413,54213,24135,24153,24513,24531$;

Domain 11 : 12345, 21345, 23145, 25431, 52431, 54231, 54321, 24315, 24351, 24531, 23415,23451 ;

Domain 12 : 12345, 21345, 23145, 23541, 25341, 52341, 53241, 53421, 54321, 12354, $21354,23154,23514,25314,52314,53214$;

Domain 13 : 12345, 21345, 23145, 25431, 52431, 54231, 54321, 23415, 23451, 23541, 25341, 52341; 
Domain 14 : 12345, 12435, 21345, 21435, 25431, 52431, 54231, 54321, 24135, 24531, 24315, 24351;

Domain 15 : 12345, 21345, 23145, 23541, 25341, 52341, 53241, 53421, 54321, 23415, 23451;

Domain 16 : 12345, 12354, 13245, 13254, 31245, 31254, 32145, 32154, 32514, 32541, $35214,35241,35421,53214,53241,53421,54321$;

Domain 17 : 12345, 13245, 31245, 32145, 32415, 32451, 35421, 53421, 54321, 34215, 34251,34521 ;

Domain 18 : 12345, 13245, 31245, 32145, 32415, 32451, 35421, 53421, 54321, 32541, $35241,53241$. 


\section{Working Paper Series in Economics}

recent issues

No. 144 Guanhao Li, Clemens Puppe and Arkadii Slinko: Towards a classification of maximal peak-pit Condorcet domains, September 2020

No. 143 Andranik S. Tangian: Using composite indicators in econometric decision models with application to occupational health, September 2020

No. 142 Ingrid Ott and Susanne Soretz: Institutional design and spatial (in)equality - the Janus face of economic integration, August 2020

No. 141 Laura Reh, Fabian Krüger and Roman Liesenfeld: Predicting the global minimum variance portfolio, July 2020

No. 140 Marta Serra Garcia and Nora Szech: Understanding demand for COVID-19 antibody testing, May 2020

No. 139 Fabian Krüger and Lora Pavlova: Quantifying subjective uncertainty in survey expectations, March 2020

No. 138 Michael Müller and Clemens Puppe: Strategy-proofness and responsiveness imply minimal participation, January 2020

No. 137 Andranik S. Tangian: Tackling the Bundestag growth by introducing fraction-valued votes, October 2019

No. 136 Susanne Fuchs-Seliger: Structures of rational behavior in economics, September 2019

No. 135 Cornelia Gremm, David Bälz, Chris Corbo and Kay Mitusch: Intermodal competition between intercity buses and trains - A theoretical model, September 2019

No. 134 Marta Serra-Garcia and Nora Szech: The (in)elasticity of moral ignorance, August 2019 